Ann. Zootech., I963, 12 (3), x73-r79.

\title{
RÉSULTATS D'UN CONTRÔLE DE DESCENDANCE PORTANT SUR LA QUALITÉ DE LA VIANDE CHEZ LE PORC
}

\author{
L. OLLIVIER et L. MESLE \\ Station de Génétique animale, \\ Centre national de Recherches zootechniques, Jouy-en-Josas (Seine-et-Oise); \\ Centre technique de la Salaison, de la Charcuterie et des Conserves de Viande.
}

\section{SOMMAIRE}

Les jambons de 346 porcs mâles et de 480 porcs femelles de race Large White (représentant les descendances de I to et $I_{4}$ I verrats respectivement) ont été soumis à des mesures de $\mathrm{pH} 24$ heures après l'abattage et à des pesées aux différentes étapes de leur transformation en "Jambon de Paris $)$.

L'étude statistique de ces données montre que les proportions de tissu gras, osseux et musculaire dans le jambon sont susceptibles d'être modifiées sous l'influence d'une sélection systématique. Par contre, une sélection portant sur le rendement du muscle lui-même à l'issue des opérations de transformation risquerait d'être d'une efficacité très limitée.

\section{INTRODUC'TION}

Des mesures nombreuses ont été proposées pour rendre compte de la qualité des viandes de porc. Pour le transformateur, le facteur essentiel de qualité est l'importance des pertes de poids que subit le muscle au cours de sa cuisson. Quant au consommateur, il est surtout sensible à la couleur et au goût des salaisons, critères qu'il est plus difficile d'apprécier objectivement.

Il a donc semblé utile de compléter le contrôle de la descendance des verrats, effectué au C.N.R.Z., par une appréciation objective de la qualité des viandes produites dans le but de savoir s'il est possible d'améliorer par sélection certains facteurs bien définis de qualité. Ainsi, de I957 à I96r, les jambons de tous les porcs de testage ont été soumis à une transformation contrôlée du type "Jambon de Paris ». Les données ainsi recueillies permettent d'évaluer l'importance des facteurs génétiques dans le rendement de cette transformation.

Annales de Zootechnie. - 1963. 


\section{MATÉRIEL, ANIMAL ET MFSURES EFFEC'TUÉES}

'Tous les jambons étudiés proviennent de porcs élevés entre le poids de $25 \mathrm{~kg}$ et celui de roo $\mathrm{kg}$ à la porcherie de testage de Jouy-en-Josas. Les animaux ont été abattus et découpés par les soins de l'abattoir du C. N. R. Z. et les jambons traités à la Charcuterie expérimentale avec le concours du C. 'T. S. C. C. V. Les diverses opérations effectuées sur les jambons ont été décrites par Mrsis et Devoyol ( 1960$)$.

Nous les rappelons ici. Ia découpe des carcasses est effectuée selon la méthode dite parisienne, 24 heures après l'abattage. Au cours de cette découpe, une note subjective de qualité de viande est attribuée à chaque jambon. Les jambons droits provenant de la découpe sont inmérliatement apportées à la Charcuterie expérimentale ou ils sont soumis a un premier "parage "qui consiste a enlever la " mouille" (partie riche en graisse et en coueme qui s"insere ent re la cuisse et l'abdomen). Une saumure leur est alors injectée, à raison de 8 p. roo de leur poids. I a composition de la sammure est la suivante :

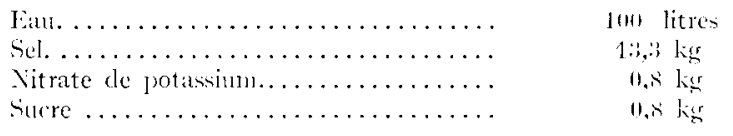

Les jambons sont alors placés dans des bacs avec tune saunure de mêne compusition que cidessus à lapuelle on ajoute environ un quart de saumure ayant déjà servi. Les hacs sont placés dans une chambre froide à une température de +4 à $+5^{\prime \prime}($.

24 jours plus tard, les jambons sont sortis de ja samure et nis il égoutler pendant 24 heures à température ambiante. Puis ils subissent un désossage et un deuxiène " parage " par lequel on s'efforce de laisser sur le jambon une quantite uniforme de gras fle couverture. Les jambons sont ensuite cuits à l'eau dans des moules en aluminimm. La cuisson est arrêtéc quand les jambons atteignent $64^{\circ} \mathrm{C}$ a cour. Aprés refroidissement ils sont placés daus une chambre froide ii $+f^{\prime \prime C}$.

Les appréciations relatives à la qualité de la viande sont de trois sortes:

$\left.I^{\circ}\right)$ une note subjective, de i à 5 ,

$\left.2^{\circ}\right)$ des pesées aux divers stadés de la transformation.

$\left.3^{\circ}\right)$ des mesures de pH, prises 24 heures après labattage au niveau de certains muscles.

lia note de qualité est toujours attribuée par la mêne personne et la note i correspond a la plus mauvaise qualité.

A partir des pesées trois rendements sont calculés. Le "rendement anatomique "tient compte des pertes qu'ent raînent les " parages zet le désosisage. Le "rendentent technologric.jue dépend essentiellement des pertes en cuisson de la portion de jambon subsistant aprés les parages et le désossage. Le " rendement final " est le simple rapport du poids final au poids init ial de jambon.

I.es mesures de pII donnent lieu au calcul de l'indes 1I, qui rejprésente la moyenne arithméticpue des pH de trois muscles : adducteur, fessier superficiel, fessier moven. ('es mesures de pl ont été prises dans le but de suppléer érentuellement aux opérations nombreuses que nécessite le contrôle de la transformation des jambons.

L'étude des différences entre races et sexes a fait l'objet des publicat ions de MesLe et al. (1959) de Dumont et Mesle (1960) et de Mesle et Drvoron ( 1960$)$.

La présente étude ne concerne que des animatix de race Large White abattus entre mars ro5i et juin I96r. Les effectifs sont de 346 mâles cast rés et 480 femelles et représentent les descendances de 140 et 44 verrats respectivement.

\section{MÉTHODES STATISTIQUES UTILISÉES}

Ia proportion des mâles et des femelles étudiés pouvant légèrement varier selon les verrats, l'analyse statistique est faite pour chaque sexe séparénent. I.es données peuvent se classcr selon un schéma hiérarchique dont les niveaux sont : l'année, le père, la mère, l'individu. Ja . ariance de chaque caractère est alors analysable en quatre composantes correspondant aux quatre niveaux 
ci-dessus, suivant une prodécure décrite par KEMPTHORNE (1957). Si on désigne les composantes ainsi obtenues par $\sigma_{\mathrm{A}}^{2}$ pour l'année, $\sigma_{\mathrm{P}}^{2}$ pour le père, $\sigma_{\mathrm{M}}^{2}$ pour la mère et $\sigma_{\mathrm{I}}^{2}$ pour l'individu, l'héritabilité peut être estimée de deux façons :

$$
\begin{aligned}
& h^{2}=\frac{4 \sigma_{\mathrm{P}}^{2}}{\sigma_{\mathrm{r}}^{2}+\sigma_{\mathrm{l}}^{2}+\sigma_{\mathrm{l}}^{2}} \\
& h^{\prime 2}=\frac{2 \sigma_{\mathrm{P}}^{2} \frac{1}{1} 2 \sigma_{\mathrm{I}}^{2}}{\sigma_{\mathrm{P}}^{2}+\sigma_{\mathrm{l}}^{2}+\sigma_{\mathrm{l}}^{2}} .
\end{aligned}
$$

Ia deuxiène estimation n'est valable que si $\sigma_{1}^{2}$ et $\sigma_{\mathrm{M}}^{2}$ ont des valeurs assez voisines, ce qui permet de supposer que l'intluence matemelle est minime. C'est l'estimation qui a été utilisée dans cette étude pour le cas des femelles. Mais, dans le cas des mâles, $\sigma_{y}^{2}$ est estimé avec une très faible précision ¿̀ cause du nombre réduit de descendants mâles par mère (en général un seul) et c'est la première estimation qui a été retemue.

Les covariances entre les caractères deux à deux sont analysées comme les variances et les corrélat ions génétiques sont obtenues de la même façon que les coeflicients d'héritabilité.

\section{RÉSULTATS}

I) Moyennes

I e tableau I montre la distribution des notes de qualité.

TABLEAU I

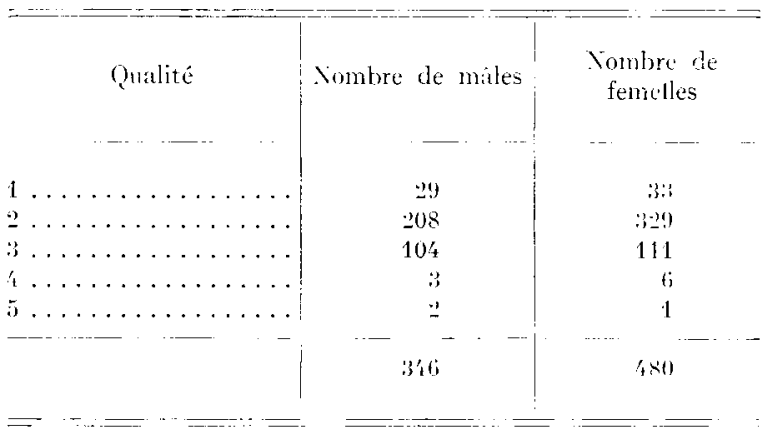

Ce tableau montre qu'environ 9I p. Ioo des viandes se situent dans les classes 2 ou 3. On pent donc dire que ces viandes se montrent assez uniformes dans leur aspect extérieur.

L,e tableau 2 indique les moyennes des mesures effectuées, ainsi que l'erreur-type attachée à chaque moyenne.

Les différences entre les sexes sont importantes pour tous les caractères saut pour le poids de la demi-carcasse. Comme tous les animaux ont été abattus au voisinage de Ioo $\mathrm{kg}$, les variations du poids de la demi-carcasse suivent étroitement celles du rendement en carcasse. Le rendement final reflète la supériorité globale des jambons des femelles qui compensent par un rendement anatomique élevé leur infériorité en rendement technologique. 


\begin{tabular}{|c|c|c|}
\hline & Mâles & Femelles \\
\hline 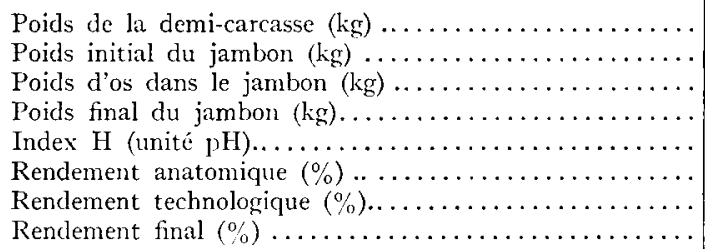 & $\begin{aligned} & 35,60 \pm 0,05 \\
& 7,59 \pm 0,01 \\
& 0,77 \pm 0,001 \\
& 4,8 \pm \pm 0,007 \\
& 5,77 \pm 0,01 \\
& 71,11 \pm 0,10 \\
& 89,70 \pm 0,12 \\
& 63,78 \pm 0,10\end{aligned}$ & $\begin{aligned} 35,54 & \pm 0,05 \\
7,81 & \pm 0,01 \\
0,79 & \pm 0,001 \\
5,04 & \pm 0,007 \\
5,74 & \pm 0,008 \\
73,16 \pm 0,10 & \pm 0,11 \\
88,20 \pm 0 & \pm 0,53 \pm 0,08\end{aligned}$ \\
\hline
\end{tabular}

\section{Il) Etude génétique}

La méthode utilisée pour estimer l'importance des facteurs génétiques dans la variation des caractères du jambon a été décrite plus haut. Il n'a pas été tenu compte dans l'analyse de variance des influences saisonnières, ni des variations dues à l'élevage dont sont issus les animaux. Ces facteurs peuvent donc éventuellement grossir la composante paternelle et par suite 1'héritabilité. Enfin les estimations d'héritabillité supposent toujours l'hypothèse de panmixie, c'est-à-dire 1'absence de consanguinité chez les individus étudiés. C'est en tenant compte de ces restrictions qu'il faut considérer les résultats du tableau 3 .

TABI,EAU 3

\begin{tabular}{|c|c|c|}
\hline Héritabilités des caractìres & Mâles & Femelles \\
\hline Poids de la demi-carcasse. & 0,71 & 0,43 \\
\hline Poids initial du jambon ......... & 1,08 & 0,35 \\
\hline Poids de l'os $\ldots \ldots \ldots \ldots \ldots \ldots \ldots \ldots \ldots \ldots \ldots \ldots$ & 1,32 & 0,93 \\
\hline Poids final du jambon...$\ldots \ldots \ldots \ldots \ldots \ldots \ldots \ldots$ & 0,81 & 0,39 \\
\hline Index II $\ldots \ldots \ldots \ldots \ldots \ldots \ldots \ldots \ldots \ldots \ldots \ldots \ldots$ & $0,9^{\prime}$ & 0,44 \\
\hline Rendement anatomique $\ldots \ldots \ldots \ldots \ldots \ldots \ldots \ldots$ & $0,9 \div$ & 0,62 \\
\hline Rendement technologique $\ldots \ldots \ldots \ldots \ldots \ldots \ldots \ldots$ & $0,3\}_{\mathrm{t}}$ & 0,09 \\
\hline 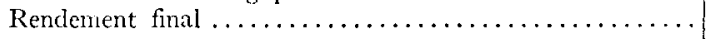 & 0,\}^{\prime}$ & 0,12 \\
\hline
\end{tabular}

Ces estimations sont affectées d'une erreur due à la taille limitée de l'échantillon. Ceci explique, en particulier, que deux des coefficients présentés ci-dessus soient supérieurs à $x$. I'évaluation exacte de l'écart-type des estimations n'est pas possible dans le cas de nos données, par suite de l'inégalité du nombre de descendants par verrat. Cependant, pour le cas, assez voisin du nôtre, de 4 femelles et 2 mâles par 
verrat, on obtient les écarts-types suivants : 0,34 pour les mâles et $0, \mathbf{I} 6$ pour les femelles, pour des valeurs d'héritabilité allant de 0,08 à 0,40 (ROBER'TSON, I059 $9^{b}$. La structure de nos données entraîne une erreur importante sur l'estimation de ce paramètre. I1 n'est donc pas possible de mettre en évidence une influence génétique sur le rendement technologique et le rendement final.

Trois caractères nous intéressent spécialement dans leurs relations entre eux : l'index de $\mathrm{pH}$, le rendement anatomique et le rendement technologique. Le tableau 4 groupe les corrélations phénotypiques (intra-année) et génétiques entre ces caractères. Les corrélations génétiques ont été estimées à partir des composantes paternelles des covariances et des variances.

TABIEAU 4

\begin{tabular}{|c|c|c|c|}
\hline & & \multicolumn{2}{|c|}{ Corrélations } \\
\hline & & Phénotypiques & Génćtiques \\
\hline $\mathrm{H} \times$ Rendement anatomique & $\begin{array}{l}\sigma^{*} \\
q\end{array}$ & $\begin{array}{l}0,06 \\
0,03\end{array}$ & $\begin{array}{l}0,2 \\
0,66\end{array}$ \\
\hline$H \times$ Rendement technologique & $\begin{array}{l}0 \\
7\end{array}$ & $\begin{array}{l}0,16 \\
0,22\end{array}$ & $\begin{array}{l}0,16 \\
0,36\end{array}$ \\
\hline Rendement anatomique $\times$ Rendement technologique & $\begin{array}{l}0 \\
+ \\
+\end{array}$ & $\begin{array}{r}0,18 \\
0,1^{\prime}\end{array}$ & $\begin{array}{r}-0,43 \\
-0,75\end{array}$ \\
\hline
\end{tabular}

\section{DISCUSSION}

Les relations entre patrimoine héréditaire et qualité de viande ne peuvent se concevoir que dans la mesure où les gènes ont une action sur les caractéristiques du tissu vivant tel qu'il se présente au moment de l'abattage. L'évolution ultérieure de ce tissu dépend partiellement de ces caractéristiques, mais de nombreux autres facteurs interviennent. La situation pourrait se représenter schématiquement de la façon suivante :

$$
\underset{\text { Gène }}{\text { Milieu } \mathrm{I}_{\text {Milieu } 2}} \text { tissu vivant }
$$

I a comparaison de trois coefficients d'héritabilité (rendement anatomique, index $\mathrm{H}$, rendement technologique) montre bien que les facteurs génétiques sont progressivement masqués à partir de l'abattage, et ce malgré les efforts faits, dans le cas présent, pour contrôler aussi bien le milieu I (conditions de l'engraissement) que le milieu 2 (conditions des opérations de saumurage et de cuisson).

La sélection pest donc être considérée comme un moyen efficace d'améliorer les 
facteurs de qualité liés à l'anatomie du jambon (en particulier la proportion de viande dans ce morceau) mais non le rendement du muscle lui-même dans les opérations de saumurage et de cuisson. Les conditions dans lesquelles ces opérations sont faites entraînent des variations d'un ordre de grandeur très supérieur aux différences proprement génétiques. Cela tient sans doute au fait que la transformation des jambons est une opération très longue (elle dure environ un mois au total). Fin outre, la méthode présente de ce fait l'inconvénient d'accroître le délai nécessaire au contrôle de la descendance.

Les différences observées entre les deux sexes pour les coefficients d'héritabilité sont systématiquement en faveur des mâles. Les erreurs d'échantillonnage ne sont donc pas les seules responsables. Ces différences pourraient être dues au nombre plus important de descendants femelles contrôlés par verrat. En effet l'abattage des femelles issues d'un verrat s'étend, en moyenne, sur une période plus longue que pour les mâles, et cela peut entraîner, pour certaines mesures, des variations intraverrat plus importantes pour les femelles et par suite une composante paternelle diminuée.

Le tableau 4 montre l'indépendance qui existe entre le rendement anatomique d'une part et le rendement technologique ainsi que les mesures de $\mathrm{pH}$ d'autre part. La corrélation entre le $\mathrm{pH}$ et le rendement technologique est positive mais faible. Quant aux corrélations génétiques, elles sont entachées d'une très grande imprécision et il serait imprudent d'en tirer des conclusions. Quoi qu'il en soit, la mesure du pH effectuée sur le jambon 24 heures après l'abattage n'apparaît pas susceptible de remplacer avantageusement la mesure de rendement technologique.

\title{
CONCLUSION
}

Des jambons provenant de 346 porcs mâles et 480 porcs femelles de race Large White ont été soumis à des mesures de $\mathrm{pH}$ et à des pesées au cours de leur transformation en "Jambon de Paris ". L'étude statistique de ces données ne permet pas de conclure à la possibilité d'améliorer génétiquement le rendement de cette transformation par une mesure directe de ce rendement.

Si l'amélioration génétique de la teneur en muscle du jambon est relativement aisée, la qualité de la viande elle-même, telle que nous avons pu la mesurer dans cette étude, dépend très peu du patrimoine héréditaire de l'individu.

$$
\text { Reç pour publication en juillet } 1963 .
$$

\section{SUMMARY}

\author{
PROGENY-TEST RESULTS ON MEAT QUALITY IN SWINE
}

The right hams from 346 male and 480 female Large White pigs were the material for this study which covered a period of time extending from March 1957 to June I96I. The animals considered were sired by $\mathrm{I}_{4} \mathrm{I}$ boars and went through the testing station located at Jouy-en-Josas. 
The following measurements were taken on the hams :

$-\mathrm{pH}, 24$ hours after slaughtering,

-. weights at various stages while processing into "Paris Ham".

Heritabilities and genetic correlations were estimated from an analysis of variance, using sire and dam components. From the estimates obtained, it was concluded that :

$\left.\mathrm{I}^{\circ}\right)$ selection can be effective in improving the proportion of meat in the ham ;

$\left.2^{\circ}\right)$ there is no prospect of genetic improvement with regard to the quality of the very muscle through the various operations of curing and cooking, if selection is directly applied to the yield of ham obtained at the end of these operations.

\section{RÉFÉRENCES BIBLIOGRAPHIQUES}

Dumont B. L., Mesle L., 1960. Caractéristiques anatomiques du membre postérieur du porc de race Piétrain. Réunion Comm. Porcine de la F. E. Z., Bruxelles.

Falconer D. S., I960. Introduction to Quantitative Genetics. Oliver and Boyd. Edinburgh et Londres. Kemphorne O., 1957. An Introduction to Genetic Statistics. John Wiley and Sons. New York.

Mesle L., Devoyod J. J., ig6o. Influence de différents facteurs sur le rendement et la qualité du "Jambon de Paris ". VI० Réunion des Instituts de Recherches sur les viandes. Utrecht.

Mesle L., Giron J., Dunont B. L., i 959. Anatomie et composition chimique du jambon. Ve Réunion des Instituts de Recherches sur les viandes, Paris.

Robertson A., I959 a. Experimental design in the evaluation of genetic parameters. Biometrics, 15, $219-226$.

Robertson A., I959 b. The sampling variance of the Genetic Correlation Coefficient. Biometrics, 15, 469-485. 